\title{
Esophagitis dissecans superficialis
}

\author{
K. K. Rawal ${ }^{1}$
}

Published online: 27 August 2015

(C) Indian Society of Gastroenterology 2015

Esophagitis dissecans superficialis (EDS) or sloughing esophagitis is an extremely rare benign illness. Endoscopically, it is characterized by sloughing of large fragments of esophageal mucosa that may be coughed up or vomited out [1]. Although an association with medications (e.g. bisphosphonates and NSAIDs), skin conditions (autoimmune bullous dermatosis), heavy smoking, and physical trauma (e.g. hot beverages, chemical irritants, and nasogastric intubation) has been reported, most cases remain unexplained and the histopathological features of EDS are not adequately described [2]. Usually asymptomatic, occasional symptoms may be dysphagia, nausea, bleeding, vomiting, heartburn and odynophagia. The endoscopic features of EDS are single or multiple translucent or opaque whitish sheets and fragments of stripped-off mucosa, involving a part or the entire esophagus (Fig. 1). In spite of a sometimes dramatic presentation, EDS is a benign condition with good prognosis and without permanent esophageal injury. A combination of acid suppression and discontinuation of precipitating medications results in complete healing [1].

K. K. Rawal

kkrawal@gmail.com

1 Department of Gastroenterology and Liver Disease, Milestone Hospital, Vidyanagar, Main Road, Rajkot 360 002, India
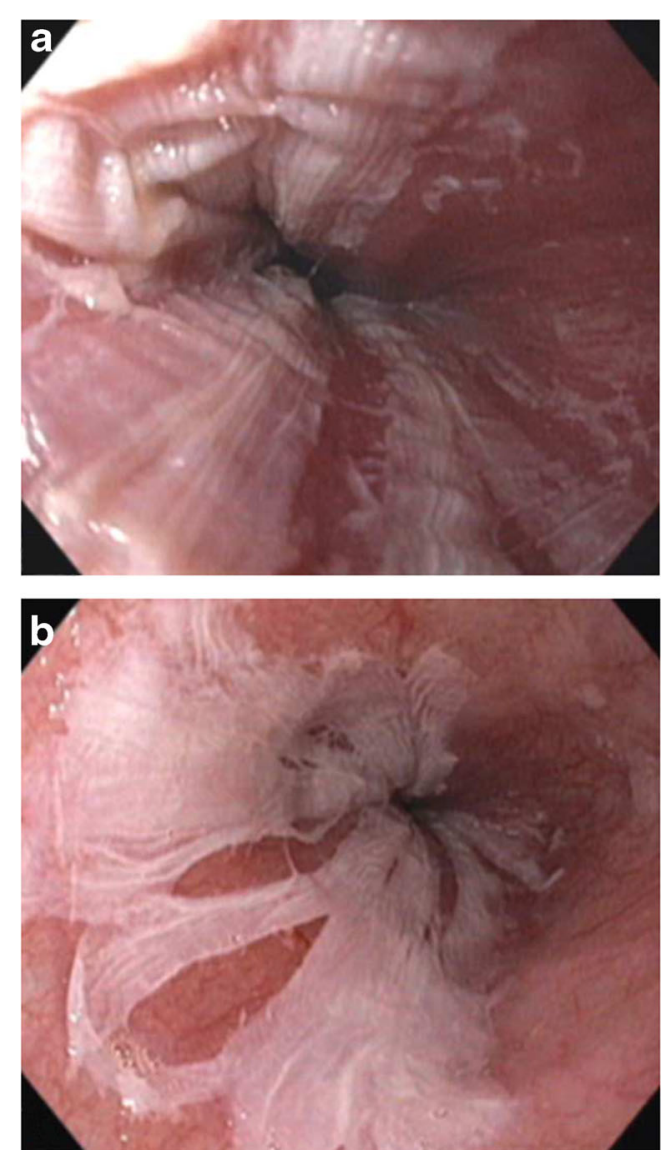

Fig. 1 Endoscopy showing esophagitis dissecans superficialis with a whitish opaque sheets of sloughed mucosa covering nearly the entire esophagus, $\mathbf{b}$ translucent and detached sheets of sloughed mucosa at places

\section{References}

1. Carmack S, Vemulapalli R, Spechler S, et al. Esophagitis dissecans superficialis (sloughing esophagitis): a clinicopathological study of 12 cases. Am J Surg Pathol. 2009;33:1789-94.

2. Purdy J, Appelman H, McKenna B. Sloughing esophagitis is associated with chronic debilitation and medications that injure the esophagus mucosa. Mod Pathol. 2012;25:767-75. 